MKG-Chirurg 2013 6 6:71

DOI 10.1007/s12285-012-0310-2

Online publiziert: 15. März 2013

(c) Springer-Verlag Berlin Heidelberg 2013

K.A. Grötz ${ }^{1,2}$

${ }^{1}$ Mund-, Kiefer- und Gesichtschirurgie, Dr.-Horst-Schmidt-Kliniken GmbH, Wiesbaden

${ }^{2}$ Kiefer- und plastische Gesichtschirurgie in der Burgstraße, Wiesbaden

\title{
Osteomyelitis und ihre Sonderformen
}

assoziierten Kiefernekrose nachhaltig geändert, da dieses Patientenkollektiv nicht mehr einer Spezialsprechstunde zugeordnet werden konnte, sondern potenziell alltäglich in der chirurgischen (und auch hauszahnärztlichen) Praxis vorstellig werden kann. Die Metapher der Medaille mit der guten und schlechten Seite drängt sich auf, denn sicherlich kann sich niemand das Ereignis einer Kiefernekrose auch nur im Entferntesten gewünscht haben, sehen wir doch gerade bei diesen Patienten, wie nachhaltig eine schwierig $\mathrm{zu}$ therapierende Erkrankung des Kieferknochens die mundgesundheitsbezogene Lebensqualität zu beeinträchtigen vermag. Operation Relevanz haben. Kie knochenschonende lokale Maßnahmen, von der Ultraschallchirurgie bis zum Alveolenerhalt vor Implantatinsertion, sind häufige Themen von Kongressen und Publikationen.

Dennoch waren noch vor wenigen Jahren Detailkenntnisse und tiefere Einsichten in die Physiologie und Pathophysiologie des Knochenstoffwechsel sowie deren Wechselwirkungen mit Allgemeinerkrankungen, lokalen und systemischen Behandlungsfolgen eher einem umschriebenen Kreis von Spezialisten vorbehalten. Entweder betrachteten diese den Knochen aus der Perspektive der Grundlagenwissenschaften oder sie waren auf die besonderen klinischen Bedürfnisse der speziellen und spezialisierten Betreuung von weniger häufigen Krankheitsbildern ausgerichtet.

Diese Situation hat sich innerhalb unseres Fachgebiets, aber insbesondere auch in der interdisziplinären Kommunikation mit einer Vielzahl anderer Facharztdisziplinen durch die 2003 bekannt gewordene Entität der bisphosphonat-

\section{》) Die Spielarten der \\ Kieferosteomyelitis \\ lassen systemische \\ Zusammenhänge erkennen}

Während die Implantologie und Parodontologie in den letzten Jahrzehnten unseren Blick zunehmend auf den lokalen Wert des Erhalts von noch so kleinen Dimensionen alveolären und periimplantären Knochenlagers geschärft hat, sensibilisierte uns die antiresorptive Therapie der Bisphosphonate (wieder) auf die übergeordneten und systemischen $\mathrm{Zu}$ sammenhänge, wie sie sich eben in den mannigfaltigen Krankheitsbilder der verschiedenen Spielarten der Kieferosteomyelitis manifestieren können. Die Einbettung des Kiefers, ja sogar der genauer umschriebenen Dimension der Kieferkämme, in ausgedehnte lokale Geschehen einer bakteriellen Osteomyelitis oder lokalen Strahlentherapiefolge, aber auch in ein primär systemisches Geschehen bei rheumatologischer Grundkrankheit oder pharmakologischer Therapiefolge wird evident.

Vor diesem aktuellen Hintergrund widmet sich diese Ausgabe dem breit gefächerten Themengebiet der Osteomyelitis des Kiefers und ihrer Sonderformen. Damit verbinden sich die Hoffnung und der Wunsch, dass es mit Interesse vom Kollegenkreis aufgenommen wird.

Ihr

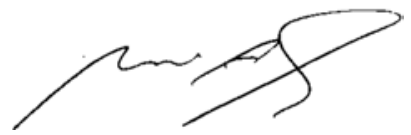

Knut A. Grötz 